Original Research Paper

\title{
The Contamination Degree of Characteristic Fungi in Jet Fuel Detected by ATP Bioluminescence
}

\author{
${ }^{1}$ Zezhen Li, ${ }^{1}$ Yun Xiong, ${ }^{2}$ Bo Chen and ${ }^{3}$ ZhenQiao Ma \\ ${ }^{I}$ Deptartment of Oil Application and Management Engineering, \\ Amry Logistics University of PLA, Chongqing, China \\ ${ }^{2}$ Sinopec Zhenhai Refining and Chemical Corps Room, Ningbo, China \\ ${ }^{3}$ Hefei Peakedness Biological Technology Co., Ltd, Hefei, China
}

\author{
Article history \\ Received: 18-04-2018 \\ Revised: $17-05-2018$ \\ Accepted: 26-05-2018 \\ Corresponding Author: \\ Yun Xiong \\ Deptartment of Oil Application \\ and Management Engineering, \\ Amry Logistics University of \\ PLA, Chongqing, China \\ Email: xyun241@126.com
}

\begin{abstract}
Adenosine Triphosphate (ATP) lysate of the characteristic fungi in jet fuel was screened and three fluorescein-luciferase systems were compared in the paper. The correlation between ATP bioluminescence and traditional plate count for measuring the number of microorganism was also investigated and then a method of detecting the contamination degree of the characteristic fungi of jet fuel by ATP bioluminescence were initially established. The results show that the effect of ATP extraction taking surfactant Benzyldimethylhexadecylammonium chloride (BAC) as the microbial lysate was optimal and the optimal concentration and the action time were $0.15 \%$ and 30 s respectively; The fluorescein-luciferase system after screening has a good detection limit, up to $10^{-15} \mathrm{~mol}$ ATP; using ATP bioluminescence and traditional plate method to measure the microbial quantity, they have good correlation. Utilizing ATP bioluminescence to detect the contamination degree of the characteristic fungi in jet fuel can shorten the test time to $10 \mathrm{~min}$, which is suitable for rapid detection and has good application prospects.
\end{abstract}

Keywords: ATP Bioluminescence, Jet Fuel, Microbial Contamination, Lysate

\section{Introduction}

Since 1930s, there are many studies on the microbial contamination of jet fuel (Graef, 2003). The existence of microorganisms may lead to blockage of aircraft fuel filters (Hill and Hill, 2008), engine damage (Hill, 2003), fuel gauge failure (Rauch et al., 2006) and oil storage equipment corrosion. The types of microbes are also different for the different storage places of fuel (Passman, 2013) and application situations (Robbins and Levy, 2005). Adenosine Triphosphate is ubiquitous in all living organisms and is used to store and transfer chemical energy, called "energy currency" (Tatsumi et al., 2003). After the organism died, ATP was rapidly degraded under the action of intracellular enzymes. Therefore, the number of viable cells can be calculated by determining the ATP concentration in the sample. ATP bioluminescence technology was produced in the mid1970s. Moyer and Henderson (1983) first proposed that the cellular ATP content can reflect the activity of cells and the number of living cells. In the same year Grb. Grönroos et al. (1983) also confirmed that this technique is a reliable and sensitive method for determining the activity of cells. McElroy (1963) first introduced the luciferase-ATP assay. In the presence of $\mathrm{Mg}^{2+}$, firefly luciferase uses D-luciferase, ATP and $\mathrm{O}_{2}$ as substrates to convert chemical energy into light energy and emit light quanta. The luminescence intensity is proportional to the ATP concentration. Therefore, the ATP concentration can be quantified by measuring luminescence intensity of the luminescent system. The ATP bioluminescence detection procedure generally includes: Sampling, ATP extraction, addition of fluorescein and luciferase, measurement of bioluminescence, determination of ATP concentration and viable cell count. In general, ATP cannot be measured without treatment of the sample. It is necessary to mix the sample with an ATP extractant to dissolve the cell membrane and cell wall to release ATP (Tedsana et al., 2013). ATP extractant is a surfactant-based reagent. The luciferase bioluminescence agent was used to measure the bioluminescence of ATP by a luminescence detector. Through the pre-determined ATP standard curve, the total ATP amount of the living bacteria can be measured. At present, people's researches on the microorganisms in jet fuel are mainly focusing on the identification and control measures of pollution strains (Andreolli et al., 2016). It 
mainly uses the traditional plate count method for the detection of microbial fuel jet pollution (Rhee, 2005), which is time-consuming and complex. Therefore, it is meaningful to establish a method to quickly detect the number of microorganisms in the fuel. ATP bioluminescence analyses the ATP number that extracted from microorganisms (Shrivastava et al., 2015) by measuring the fluorescence intensity of the reaction system and reacts the number of microorganisms indirectly (Shibata et al., 2018), where the whole detection process can be finished in a few minutes (Hu et al., 2016). Presently, ATP bioluminescence has been widely used in food hygiene, environmental monitoring and other aspects (Wang et al., 2015); however, little research has been done on the degree of microbial contamination in jet fuel. Ferrari et al. (1998) performed microbial colony analysis on jet fuel samples by culture method and considered that the main contaminating fungi in jet fuel were Amorphotheca and Aspergillus. The experiment begins with the enrichment of microorganisms in jet fuel to the optimal ATP extraction method of microbial samples and the screening of fluorescei-luciferase reaction system; the detection method of microbial contamination degree in jet fuel is established primarily and compared with the traditional plate count method, which can be replaced in a certain range.

\section{Materials and Methods}

Materials and instruments. Oil sample: No. 3 military jet fuel, which is provided by Sinopec Zhenhai Refining and Chemical Company. Test strains: Amorphotheca resinae, Penicillium restrictum; the bacteria in the laboratory are isolated from the jet fuel; Escherichia coli (CICC 23796). Medium: Sabouraud medium (100 mL distilled water, $4 \mathrm{G}$ glucose, $1 \mathrm{~g}$ peptone and $1.5 \mathrm{~g}$ agar powder), LB medium (100 $\mathrm{mL}$ distilled water, $1 \mathrm{~g}$ peptone, $0.5 \mathrm{~g}$ yeast extract, $0.5 \mathrm{~g}$ sodium chloride and $2 \mathrm{~g}$ agar powder), sterilization for $20 \mathrm{~min}$ at $121^{\circ} \mathrm{C}$. Reagent: CTAB, BAB, BAC and SDS were purchased from Shanghai Aladdin Biochemical Polytron Technologies Inc.; standard ATP was purchased from Shanghai Promega Biological Products Co. Ltd.; Fluorescein-luciferase was purchased from A, B and C companies in China respectively. Instruments: The optical absorption eliasa VersaMax with single function from Molecular Devices Corporation (MDC); HY-LiTE Jet A1 Fuel Test from Merck company; fast and automatic high pressure sterilizer TOMY SX from Japan; vertical flow clean bench ESCO OptiMair and SPX-150 incubator from Beijing Houhui experimental instrument co.

Screening of fluorescein luciferase system. The standard ATP $\left(10^{-2} \mathrm{~mol} / \mathrm{L}\right)$ using ATP buffer was diluted to $10^{-8}, 3 \times 10^{-9}, 10^{-9}, 3 \times 10^{-10}, 10^{-10}$ and $3 \times 10^{-11} \mathrm{~mol} / \mathrm{L}$ respectively; The standard ATP solution $50 \mu \mathrm{L}$ with each concentration were put into three different fluorescein luciferase systems respectively for luminous value measure. The standard ATP is diluted with sterile water and the amount of fluorescein-luciferase used in each experiment is also consistent. Because the detection of fluorescence intensity is greatly affected by the operation, the experiment for each dilution is repeated in parallel three times and averaged.

Screening of ATP lysate. $1 \mathrm{~mL}$ fungal liquid was added to $1 \mathrm{~mL} 0.1 \%$ of $\mathrm{BAC}, \mathrm{BAB}, \mathrm{CTAB}$ and SDS solution respectively; $50 \mu \mathrm{L}$ reaction solution after $1 \mathrm{~min}$ reaction was added to the fluorescein-luciferase reaction system screened before for luminous value measure and sterile water was used as blank control. The lysate and the equipment used in the experiment were sterilized at $121^{\circ} \mathrm{C}$ and high pressure for $20 \mathrm{~min}$ prior to use. Detection of fluorescence intensity was repeated in parallel 3 times and averaged.

The optimum concentration and reaction time of BAC extracting ATP. Different concentrations of BAC lysate were allocated and $1 \mathrm{~mL}$ mold solution was added to $1 \mathrm{~mL}$ BAC lysate with different concentrations respectively; after concussion for $1 \mathrm{~min}, 50 \mu \mathrm{L}$ reaction liquid are added to the fluorescein-luciferase system to measure the luminous value. The optimum extraction time was selected by the same method after determining the optimum concentration. The reaction time of the sample in the fluoresceinluciferase system needs to be taken into account in the action time of the BAC and the fluorescence intensity should be averaged 3 times in parallel.

The correlation between ATP bioluminescence and plate count method. Mold was added to PDA slope and 5 $\mathrm{mL}$ sterile water was added to it after $7 \mathrm{~d}$ cultivation at $26^{\circ} \mathrm{C}$ The spore on the slope was gently scraped; $10 \mathrm{~mL}$ spores suspension was obtained after shaking treatment and then stored at $4{ }^{\circ} \mathrm{C}$ after cell count. The suspension of mold spores was diluted to different gradients by aseptic water, which was detected utilizing traditional plate count and ATP bioluminescence respectively and the correlation of them was analyzed. The spore needs to be observed under a microscope, according to its shape and characteristics to determine the corresponding mold.

\section{Results}

\section{Screening Results of Fluorescein-Luciferase System}

The screening results of fluorescein-luciferase system are shown in Fig. 1. The correlation of type $\mathrm{C}$ in the three kinds of fluorescein-luciferase systems is higher than type A and the linear range of it is wider than type B. Through regression analysis, the one-dimensional linear regression equation obtained is $\mathrm{Y}=0.8080 \mathrm{X}+8.3884, \mathrm{R}^{2}=$ 0.9889 ; in the range of $10^{-8} \sim 3 \times 10^{-11} \mathrm{~mol} / \mathrm{L}$, fluorescence intensity has a good linear relationship with ATP concentration that bioluminescence can be used to measure the degree of microbial contamination in jet fuel. 


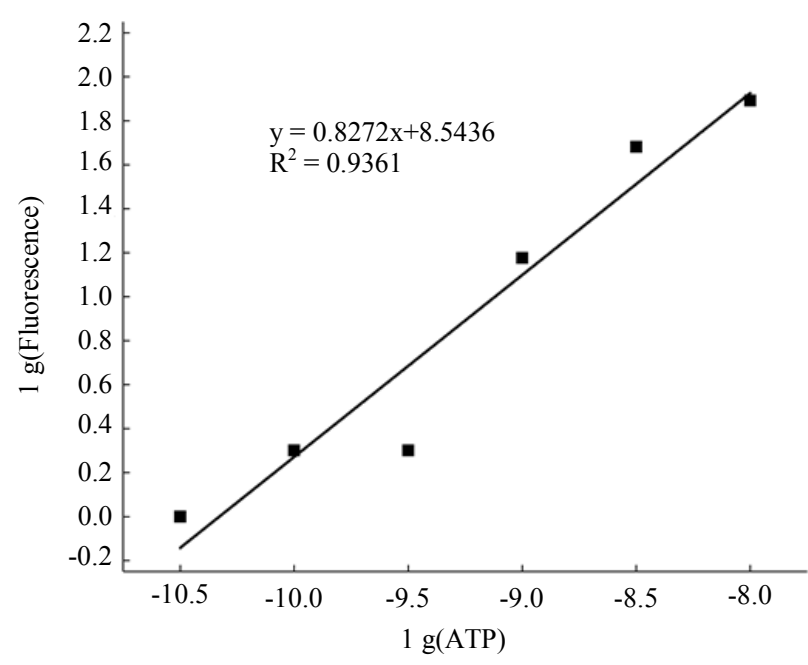

(A)

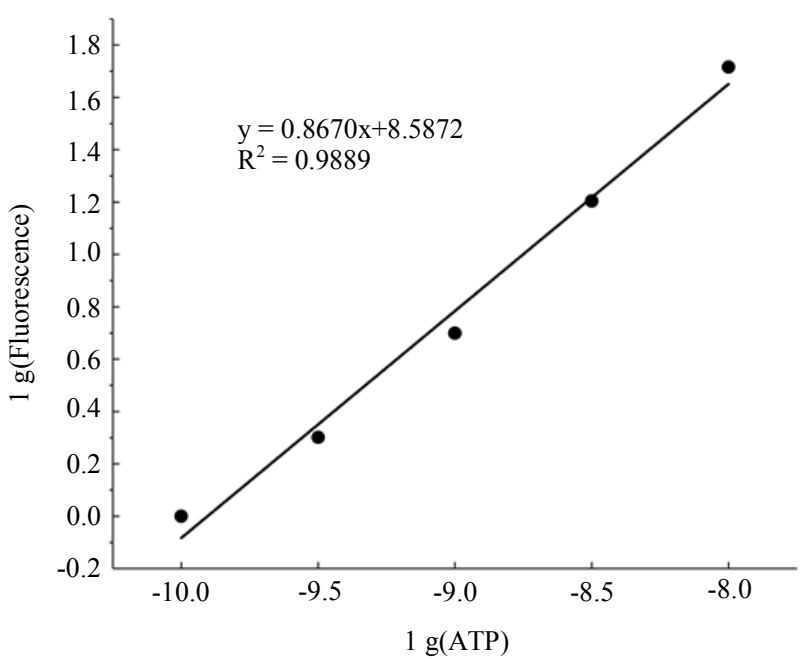

(B)

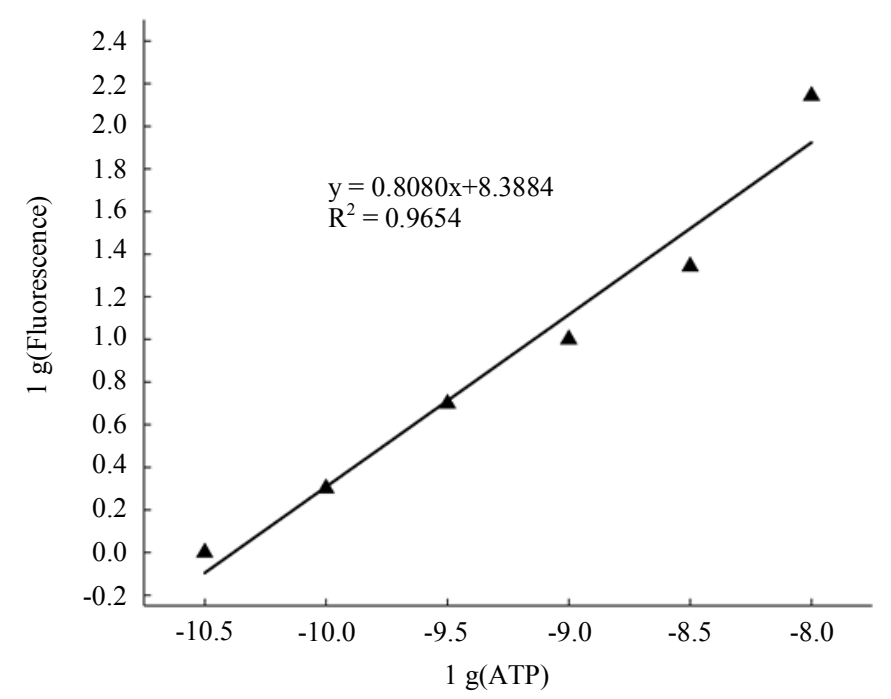

(C)

Fig. 1: ATP concentration and the standard curve of luminescence intensity of fluorescein-luciferase A, B and C

\section{ATP Screening Results of ATP Lysate}

The effects of each ATP lysate on Amorphotheca resinae and Penicillium restrictum were shown in Table 1.

It can be seen in Table 1 that the effects of the surfactant lysate $\mathrm{BAB}, \mathrm{BAC}$ and $\mathrm{CTAB}$ are optimal, which can significantly enhance the fluorescence intensity. Among them, BAC has the best effect with lower virulence and convenient use and is a good choice for cracking solution.

\section{The Optimum Concentration and Time of Lysate}

The screening results of lysate concentration are shown in Fig. 2A. We can see that the fluorescence intensity first increases and then decreases with the increase of BAC concentration and the high BAC concentration would have inhibitory effect on fluorescence system. The fluorescence intensity is the maximum when the BAC concentration is $0.15 \%$. The reaction time of lysate is shown in Fig. 2B; it can be seen that the fluorescence intensity is reduced rapidly with time growth, which may be determined by the reaction process of fluorescence system and ATP. Therefore, the optimum reaction condition of cracking process was $0.15 \%$ BAC with 30 s action. It is speculated that the main reason for the decrease of the fluorescence intensity with time is that the stability of the fluorescein-luciferase system is not strong, the reaction rate of the enzyme is unstable and the decay rate of the fluorescence intensity is too fast. The second reason may be that the detection stability of the fluorescence detector is insufficient, because the fluorescence intensity of ATP itself is weak and it is very easy to receive the influence of other light sources and the sealability of the instrument is very important. 
Table 1: ATP cracking effect of different lysates

\begin{tabular}{|c|c|c|}
\hline \multirow[b]{2}{*}{ Lysate } & \multicolumn{2}{|c|}{ Fluorescence intensity /RLU } \\
\hline & Amorphotheca resinae & Penicillium restrictum \\
\hline Sterile water & $20 \pm 2$ & $27 \pm 3$ \\
\hline $\mathrm{BAB}$ & $1326 \pm 17$ & $1569 \pm 23$ \\
\hline $\mathrm{BAC}$ & $1473 \pm 17$ & $1754 \pm 29$ \\
\hline CTAB & $721 \pm 10$ & $1178 \pm 20$ \\
\hline SDS & $32 \pm 4$ & $67 \pm 9$ \\
\hline
\end{tabular}

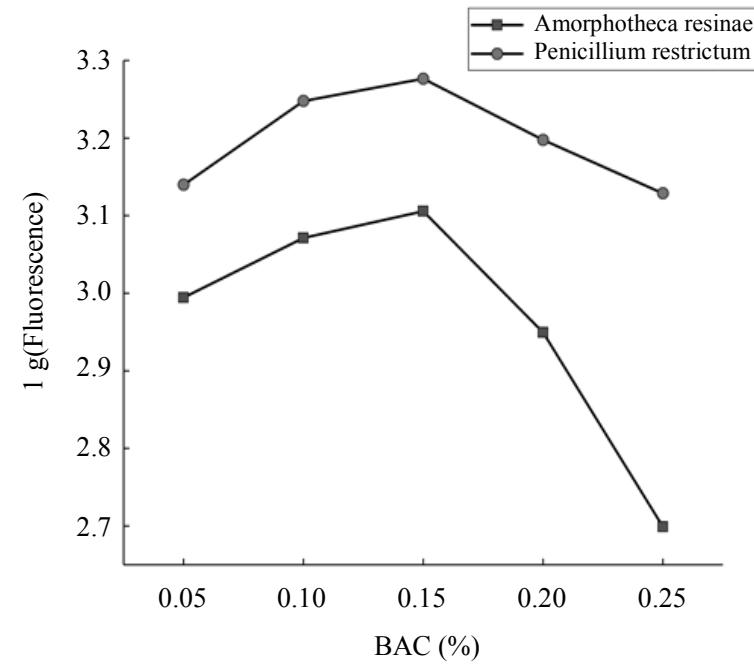

(A)

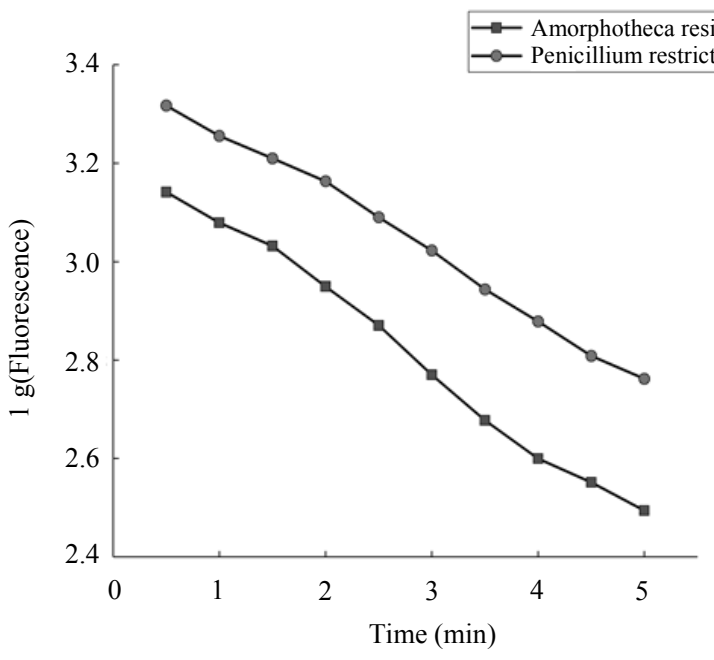

(B)

Fig. 2: The optimum concentration and time of BAC lysate

\section{The Correlation Between ATP Bioluminescence and Traditional Plate Count Method}

The concentration of characteristic fungi of jet juel Amorphotheca resinae and Penicillium restrictum was diluted to different gradients and the number of them was measured by ATP bioluminescence and plate count method. The result is shown in Fig. 3. We can see from Fig. 3, in the test range, the results of the fungi were linear correlation and the correlation coefficient was above 0.99 , which indicates that ATP bioluminescence measuring the number of fungi has strong correlation with traditional plate count method and can be used to detect the contamination degree of microorganisms in jet fuel.

\section{Discussion}

In this paper, the ATP bioluminescence assay is used to detect the degree of microbial contamination in jet fuels. Two kinds of fungi in jet fuel were selected as experimental objects (Gaylarde et al., 1999). First, select three luciferin-luciferase products on the market as the object of the study. After diluting the standard of ATP reagent to a certain percentage, screen the product by examining the linear relationship between fluorescence intensity and ATP concentration. The paper screens the fluorescein-luciferase systems of $\mathrm{A}, \mathrm{B}$ and $\mathrm{C}$ companies in China, the results show that product $\mathrm{C}$ is in the range of $10^{-8} \sim 3 \times 10^{-11} \mathrm{~mol} / \mathrm{L}$, whose fluorescence intensity has a good linear relationship with ATP concentration and the bioluminescence can be used to detect the microbial contamination degree in jet fuel. However, under actual use conditions, the amount of microorganisms in jet fuel itself is extremely small and there is a loss during the collection of microorganisms and the release of ATP. It is also necessary to consider the detection error of the instrument, so it needs to be more sensitive and lower luciferin-luciferase luminescence system. There are many ways to extract ATP and different methods have different effects on the luminous intensity. The ATP extractant mainly has the following requirements: It can rapidly kill live cells and break up and dissolve cell membranes; denature and deactivate ATP hydrolyzing enzymes or invertases; fully release intracellular ATP into the reaction system; does not interfere or rarely interfere with the luciferin-luciferase system. Four commonly used microbial lysate were screened and the best action concentration and time were further studied. The results showed that $0.15 \%$ BAC after 30 s action could significantly increase the fluorescence intensity. 


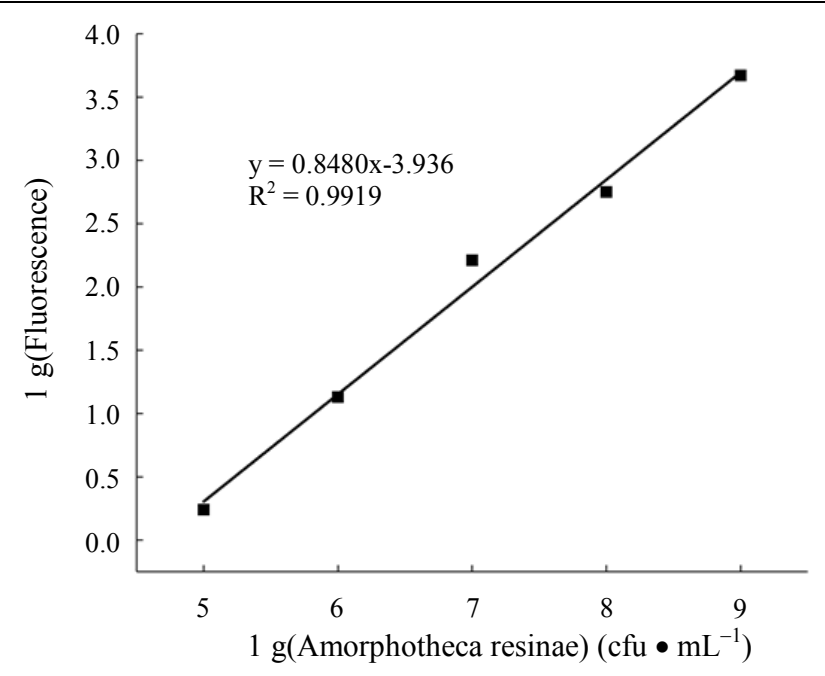

(A)

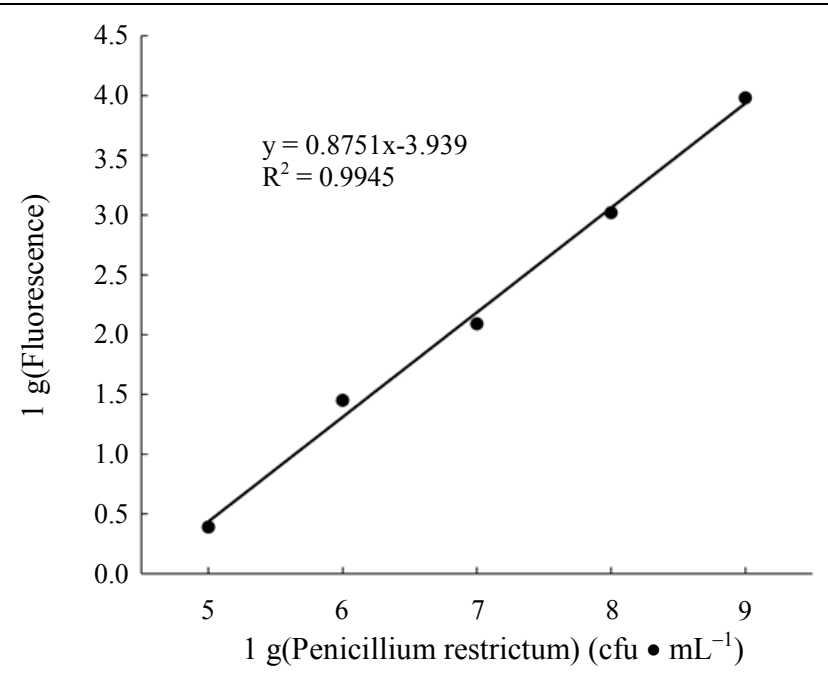

(B)

Fig. 3: The relationship between the number of characteristic fungi and the luminescence intensity of ATP

The measurement of the amount of microorganisms in jet fuel is generally based on the traditional culture method, but the culture method can not produce some microorganisms and the operation is complicated and time consuming. It cannot meet the requirements of rapid detection, especially for military jet fuel. Strict and rapid detection methods. The number of characteristic fungi was detected by ATP bioluminescence and traditional plate culture and the correlation of the two methods was studied. The results show that the two methods have good correlation. In a certain range, the ATP bioluminescence method can be used to detect the pollution degree of the characteristic fungi. The ATP bio-fluorescence assay is used to detect the degree of contamination of characteristic microorganisms in jet fuel. The time of the entire method is very short and the detection process is relatively simple. It is very suitable for on-site detection. The next step is to improve the stability of the fluorescence instrument and the fluorescein-luciferase system. In summary, the ATP bioluminescence assay can be used to detect the degree of microbial contamination of jet fuel, but at the present stage it also faces the problem of low accuracy and high detection limits, but it can be used as a preliminary detection method if there is suspected contamination. The situation is specifically determined by the combined culture method.

\section{Conclusion}

This article uses ATP bioluminescence to detect the degree of microbial contamination in jet fuel. A highefficiency luciferin-luciferase system was screened through experiments and the optimal concentration and action time of the ATP lysate were examined. The resulting detection method can quickly detect the order of magnitude of microbes in jet fuel within 10 min with a detection limit of $10^{-15} \mathrm{~mol}$ ATP. This method can be applied to the rapid detection on the spot. If the activity of the enzyme can be improved, the accuracy of the detection can be further improved.

\section{Acknowledgement}

We are grateful to thank the sinopec zhenhai refining and chemical corps room for their cooperation and technical guidance during the study period. We are also grateful thankful to Sr. Ma for his help during the writing period.

\section{Author's Contributions}

Zezhen Li: He designed the research plan and organized the study.

Yun Xiong: He participated in all experiments, coordinated the data-analysis and contributed to the writing of the manuscript.

Bo Chen: He participated to collect the materials related to the experiment.

ZhenQiao Ma: It is contributed to get support from the supporters.

\section{Conflict of Interest}

The authors declare that they have no competing interests. The corresponding author affirms that all of the authors have read and approved the manuscript.

\section{Ethics}

The authors declare that they had no conflict of interest. 


\section{References}

Andreolli, M., N. Albertarelli, S. Lampis, P. Brignoli and N.S. Khoei et al., 2016. Bioremediation of diesel contamination at an underground storage tank site: A spatial analysis of the microbial community. World J. Microbiol. Biotechnol., 32: 6-6. DOI: $10.1007 / \mathrm{s} 11274-015-1967-2$

Ferrari, M.D., E. Neirotti and C. Albornoz, 1998. Occurrence of heterotrophic bacteria and fungi in an aviation fuel handling system and its relationship with fuel fouling. Revista Argentina Microbiol., 30: 105-114.

Gaylarde, C.C. F.M. Bento and K. Joan, 1999. Microbial contamination of stored hydrocarbon fuels and its control. Brazilian J. Microbiol., 30: 331-345. DOI: $10.1590 / \mathrm{S} 0001-37141999000100001$

Graef, H.W., 2003. An analysis of microbial contamination in military aviation fuel systems. MSc Thesis in Engineering and Environmental Management, Department of System and Engineering Management.

Grönroos, M., J. Mäenpää, A.L Nieminen and L. Kangas, 1983. 548 Correlation of steroid receptor contents with medroxyprogesterone and tamoxifen effects in endometrial cancer assayed by, barin vitro ATPbioluminescence method. J. Steroid Biochem., 19: 1-194. DOI: 10.1016/0022-4731(83)92049-6

Hill, E.C. and G.C. Hill, 2008. Microbial contamination and associated corrosion in fuels, during storage, distribution and use. Adv. Mater. Res., 38: 257-268. DOI: $10.4028 /$ www.scientific.net/AMR.38.257

Hill, T., 2003. Microbial growth in aviation fuel. Aircraft Eng. Aerospace Technol., 75: 497-502. DOI: $10.1108 / 00022660310492582$

$\mathrm{Hu}, \mathrm{T}$., W. Na, X. Yan and X. Su, 2016. Sensitive fluorescence detection of ATP based on host-guest recognition between near-infrared $\beta$-cyclodextrincuins2 qds and aptamer. Talanta, 165: 194-200. DOI: 10.1016/j.talanta.2016.09.064

Mcelroy, W.D., 1963. [63] Crystalline firefly luciferase: lh $2,+$ atp $\rightleftarrows \mathrm{lh} 2 \cdot$ amp + pp lh $2 \cdot$ amp + o $2, \rightarrow$ l-amp + light + h 2 o. Methods Enzymol., 6: 445448. DOI: 10.1016/0076-6879(63)06203-0
Moyer, J.D. and J.F. Henderson, 1983. Nucleoside triphosphate specificity of firefly luciferase. Anal. Biochem., 131: 187-189. DOI: 10.1016/0003-2697(83)90152-5

Passman, F.J., 2013. Microbial contamination and its control in fuels and fuel systems since $1980-$ a review. Int. Biodeteriorat. Biodegradat., 81: 88-104. DOI: 10.1016/j.ibiod.2012.08.002

Rauch, M.E., H.W. Graef, S.M. Rozenzhak, S.E. Jones and C.A. Bleckmann et al., 2006. Characterization of microbial contamination in United States air force aviation fuel tanks. J. Indus. Microbiol. Biotechnol., 33: 29-36. DOI: 10.1007/s10295-005-0023-x

Rhee, I.S., 2005. Microbiogical contamination in jp-8 fuel. Aviat. Fuels.

Robbins, J.A. and R. Levy, 2005. A review of the microbiological degradation of fuel.

Shibata, M., G. Elmasry, K. Moriya, M.M. Rahman and Y. Miyamoto et al., 2018. Smart technique for accurate monitoring of ATP content in frozen fish fillets using fluorescence fingerprint. LWT Food Sci. Technol. DOI: 10.1016/j.lwt.2018.02.026

Shrivastava, S., I.Y. Sohn, Y.M. Son, W.I. Lee and N.E. Lee, 2015. Real-time label-free quantitative fluorescence microscopy-based detection of atp using a tunable fluorescent nano-aptasensor platform. Nanoscale, 7: 19663-19672. DOI: $10.1039 / \mathrm{C} 5 \mathrm{NR} 05839 \mathrm{~B}$

Tatsumi, T., J. Shiraishi, N. Keira, K. Akashi and A. Mano et al., 2003. Intracellular ATP is required for mitochondrial apoptotic pathways in isolated hypoxic rat cardiac myocytes. Cardiovascular Res., 59: 428-440.

DOI: $10.1016 / \mathrm{S} 0008-6363(03) 00391-2$

Tedsana, W., T. Tuntulani and W. Ngeontae, 2013. A highly selective turn-on ATP fluorescence sensor based on unmodified cysteamine capped CDS quantum dots. Anal. Chim. Acta, 783: 65-73. DOI: 10.1016/j.aca.2013.04.037

Wang, X., Z. Zhang, X. Ma, J. Wen and Z. Geng et al., 2015. Real-time fluorescence assays of alkaline phosphatase and ATP sulfurylase activities based on a novel PPI fluorescent probe. Talanta, 137: 156-160. DOI: 10.1016/j.talanta.2015.01.028 